\title{
A Novel Bioreactor to Study the Dynamics of Co-culture Systems
}

\author{
M. H. Kim ${ }^{1}$, M. Liang ${ }^{1}$, Q. P. He ${ }^{2}$ and J. Wang ${ }^{1}$
}

(1) Department of Chemical Engineering, Auburn University, Auburn, AL 36849, USA

(2) Department of Chemical Engineering, Tuskegee University, Tuskegee, AL 36088, USA

Correspondence: Jin Wang, Auburn University, 212 Ross Hall, Auburn AL 36849, USA

Tel: 1-334-844-2020. Fax: 1-334-844-2063. Email: wang@ auburn.edu

\begin{abstract}
Co-culture strategy has drawn increasing interest in the last a few years to ferment the mixture of glucose and xylose or lignocellulosic hydrolysate. However, existing research has been mostly qualitative which mainly examined the ethanol production performance of the co-culture system such as final ethanol yield and productivity, with little or no attempt made to understand the dynamic interactions between the two microbes. This is partially due to the difficulties associated with monitoring and control of co-culture systems. In this work, we developed a bioreactor and associated protocols and control strategies to facilitate quantitative and systematic study of co-culture systems. In particular, the reported equipment, operation protocols and control strategies can deliver chemostat operation under controlled stable operation conditions by achieving stable oxygen utilization rates at various levels. In addition, the developed membrane-separated co-culture bioreactor enables independent control of the dissolved oxygen (DO) levels in each chamber, and easy tracking of individual biomass of each strain. The new protocol is a dual continuous/pseudo-continuous operation mode, which allows the control of constant biomass by adjusting the operation time of each mode, dilution rate and feed concentration. Experimental results on the co-culture system of Saccharomyces cerevisiae and Scheffersomyces stipitis are provided to demonstrate the capabilities of the developed coculture bioreactor. With the developed equipment and protocol, simultaneous and complete
\end{abstract}


utilization of both glucose and xylose was achieved around 70 hours into the experiments we conducted, and was maintained as long as 800 hours. Such complete utilization can be maintained even longer if desired. In addition, different OUR conditions (ranging 0.0036 $0.0045 \mathrm{mmolO}_{2} / \mathrm{gDCW} / \mathrm{hr}$ ) were tested under controlled chemostat. Under the different operation conditions tested, ethanol yields and conversion rates varied in the range of 0.12$0.44 \mathrm{~g} / \mathrm{g}$ and $0.22-1.95 \mathrm{gEtOH} / \mathrm{L} / \mathrm{hr}$, respectively, which are in line with results reported in the literature.

Keywords: co-culture; bioreactors; control; bioprocess monitoring; fermentation; ethanol 


\section{Introduction}

Lignocellulosic biomass is an attractive, sustainable, and abundant carbon source for ethanolic fermentation process. However, the lack of microorganisms which can efficiently ferment all sugars derived from lignocellulosic biomass is one of the key barriers in industrializing lignocellulosic ethanol process. To overcome this barrier, numerous microorganisms have been constructed in the past few decades to ferment the mixture of glucose and xylose, the dominant hexose and pentose from lignocellulosic hydrolysate [13]. As an alternative, co-culture strategy has drawn increased attention for simultaneous conversion of glucose and xylose. Table 1 summarizes recent attempts in using co-culture systems for glucose/xylose or lignocellulosic hydrolysate fermentation with two older continuous co-culture studies. Existing results show that co-culture is a promising way to ferment mixed glucose and xylose for ethanol production, especially in reducing fermentation time and improving ethanol productivity. However, existing research mainly examined the ethanol production performance of the co-culture system such as final ethanol yield and productivity, with little or no attempt made to understand the dynamic interactions between the two microbes. In addition, major challenges associated with the co-culture strategy have not been adequately addressed, which include the incompatible oxygen conditions required by two microbes for optimal ethanol production, catabolite repression of xylose utilization when glucose is present, and the low ethanol tolerance of xylose-fermenting microorganisms [4-7].

In this work, we developed a new bioreactor and a new protocol to address some of the above-mentioned challenges, which allow the systematic investigation of the dynamic 
properties of a co-culture system: Saccharomyces cerevisiae and Scheffersomyces stipitis. The new equipment is a membrane-separated co-culture bioreactor which enables the independent control of the dissolved oxygen (DO) levels in each chamber, and the easy tracking of the biomass of each strain. The new protocol is a dual continuous/pseudocontinuous operation mode, which allows the control of constant biomass by adjusting the operation time of each mode, dilution rate and feed concentration. It is worth noting that membrane-separated bioreactors have been reported in different applications and they have many benefits, including obtaining high cell density culture, easy control or separation of cells, retention of cellulase, etc. [8-12]. However, one of the major issues with these systems is the membrane fouling due to the adhesion of cells on the filtration surface. In addition, some systems were found to be difficult to control the specific OUR [9]. In comparison, the system developed in this work could keep running for several months under controlled OUR and chemostat condition.

\section{Materials and Methods}

\subsection{Microorganisms and culture media}

The strains used in this study were S. cerevisiae D5A and S. stipitis CBS 5773 which were obtained from Y.Y Lee, Auburn University and ATCC, respectively. The frozen stock and culture media were the same as in Liang et al. [9]. The sugar concentrations of feed medium for pseudo-continuous fermentation were varied based on the operational conditions.

\subsection{Reactor design and development}

The schematic diagram and actual set-up of the developed membrane-separated bioreactor are shown in Figure 1. The body of the bioreactor was constructed using 
polycarbonate, which consists of two chambers. By placing a microporous filter membrane (Biodyne ${ }^{\circledR}$ Plus, Pall Corporation, NY) between the two chambers, the two strains are separated by the membrane while various metabolites can exchange between the two chambers. The Biodyne ${ }^{\circledR}$ plus membrane is a nylon membrane which consists of Nylon 6,6 cast as a symmetric microporous membrane (pore size $0.45 \mu \mathrm{m}$ ) on non-woven polyester support and is sterilized together with the bioreactor using autoclave. The propellers of the agitators are driven by external magnetic fields; $\mathrm{pH}$ and temperature are under automatic feedback control (not shown in Figure 1a). This two-chamber configuration enables us to easily monitor and control the biomass development of individual strains during fermentation. It also allows the independent control of different oxygen conditions for each strain by adjusting nitrogen and air gas flow rates to each chamber. In addition, by feeding the substrates into $S$. cerevisiae chamber, glucose can be completely consumed by $S$. cerevisiae, leaving only xylose reaching S. stipitis chamber. Therefore, the catabolite repression of xylose utilization can be alleviated.

\subsection{Dual-mode operation}

Pseudo-continuous operation is characterized by continuous nutrient feeding and continuous cell-free broth withdrawal, which can be achieved through a cell retention module to keep cells from effluent. The biggest challenge in pseudo-continuous operation is maintaining the stability and effectiveness of cell filtration module. Due to the continuous buildup of cells on the filtration surface, commercial filtration modules usually have limited operation time even with back-flash implemented periodically [9] . To address this difficulty, we have developed a cell retention module in-house, with the filtration surface located right against the propeller blades as shown in Figure 1a. In this way the 
membrane (Supor800, pore size $0.8 \mu \mathrm{m}$, Pall Corporation, NY) surface is constantly cleaned through the shear stress generated by agitation, which allowed prolonged pseudocontinuous fermentation for months [13]. Because S. stipitis grows very slowly under oxygen limited condition, "wash-out" is often resulted under continuous operation. With pseudo-continuous mode as an option, the biomass of S. stipitis can be controlled by the dual-mode operation, which combines pseudo-continuous operation (for biomass accumulation) and continuous fermentation (for biomass maintaining or reduction). The operation time of each mode depends on the cell growth rate and dilution rate, and the detailed calculation of the operation time for each mode can be found in Appendix A.

\subsection{Independent control of oxygen supply and monitoring of oxygen utilization rate}

The conversion efficiency of xylose to ethanol by S. stipitis is highly influenced by oxygen utilization rate (OUR) [14-16]. In order to quantitatively study the effect of OUR on the fermentation performance and by-product secretion, maintaining stable OUR at different levels to achieve chemostat is necessary. It should be noted that OUR cannot be directly controlled as one cannot control how much oxygen cells will pick up, it can only be manipulated through controlling the composition of the feeding gas into the system. To achieve various stable OUR conditions, feed gas with different oxygen compositions but constant total gas flow rate has to be delivered to the system. Our tests showed that the standard gas mixing component equipped with New Brunswick BioFlo 115 cannot deliver the desired stable feeding gas, as the total gas flow rate changes noticeably when the ratio between air and nitrogen is adjusted. To address this, we have developed and tested a gasmixing apparatus by adding a buffer tank, which can deliver the desired stable feeding gas 
as confirmed by GC measurements. Alternatively, stable feeding gas with different oxygen concentrations can be achieved with mass flow controllers.

The volumetric mass transfer coefficient $\left(k_{L} a\right)$ of oxygen is an important factor for fermentation process which can be affected by many factors such as cell density, broth viscosity, etc. [17]. Based on our studies, we found that $k_{L} a$ value varies noticeably during the course of an experiment. Therefore, one cannot rely on a measured $k_{L} a$ under a fixed condition to estimate the oxygen utilization rate (OUR) during an experiment, which describes how much oxygen the cells actually picked up. To address this challenge, we used mass balance to calculate OUR by measuring oxygen concentration in the inlet gas and off-gas. The measured $k_{L} a$ is used as a guide to determine the inlet gas composition in order to achieve desired OUR. It is worth noting that the obtained OUR will be close to the target, but will almost never be exactly on target, as the actual OUR is determined by many factors, such as the cell density or the total number of cells in the reactor, the viability and metabolic state of the cells, and the oxygen supply.

\subsection{Ethanol tolerance test}

The ethanol tolerance of cells was evaluated by comparing their survival rates after 2 hours exposure to fermentation media containing 0 (control), 30, 60, 90, $120 \mathrm{~g} / \mathrm{L}$ ethanol for $S$. cerevisiae, and 0 (control), 15, 30, 45, $60 \mathrm{~g} / \mathrm{L}$ ethanol for S. stipitis, following a modified procedure of Zhou et al. [18]. In this work, the unadapted cells means cells sampled directly from pre-culture and the adapted cells means cells sampled from 2 weeks of pseudo-continuous fermentation after inoculation of pre-cultured cells. Both unadapted and adapted cells were cultured in the pre-culture medium for 24 hours and harvested in 
mid-exponential growth phase to eliminate possible effect of different physiological states on cells' ethanol tolerance.

\subsection{Experimental procedure}

All experiments were conducted under temperature of $25^{\circ} \mathrm{C}$ and $\mathrm{pH}$ of 5.0. The $\mathrm{pH}$ was maintained at 5.0 by automatic addition of $1.0 \mathrm{~N} \mathrm{NaOH}$. The membrane-separated bioreactor is set up in the way that for each chamber, its effluent can be withdrawn either with or without cell retention, which enables the dual continuous/pseudo-continuous operation. With this setup, three operation modes can be implemented in the bioreactor: batch, pseudo-continuous and continuous. In general, each experiment consists of two stages: cell growth and fermentation. The purpose of cell growth stage is to achieve the desired cell density of both strains for fermentation stage, while the fermentation stage allows the examination of the effect of different factors on the fermentation performance.

Cell growth stage can be implemented through either batch operation or pseudocontinuous operation. After inoculation, both S. cerevisiae and S. stipitis grow under oxygen limited-condition by purging a mixture of air $(156 \mathrm{~mL} / \mathrm{min})$ and nitrogen (40 $\mathrm{mL} / \mathrm{min}$ ) gas at the fixed total flow rate of $196 \mathrm{~mL} / \mathrm{min}$ at atmospheric pressure. Such oxygen condition allows relatively fast cell growth while producing ethanol, which promotes cell adaption to higher ethanol concentration. The fermentation stage is implemented through dual pseudo-continuous/continuous operation, depending on the cell growth rate under different oxygen conditions. During this stage, the oxygen condition for the S. cerevisiae chamber is maintained at anaerobic by feeding nitrogen gas only, while different oxygen conditions (i.e., different OUR) were tested for S. stipitis chamber through manipulating the air to nitrogen ratio of the feeding gas. It should be noted that it is 
necessary to maintain constant biomass density under each oxygen condition in order to achieve stable OUR through maintaining stable oxygen supply.

\subsection{Analytical methods}

For the analytical procedures, the concentrations of glucose, xylose, xylitol, glycerol, acetic acid and ethanol and the cell density of each strain were analyzed as described in Liang et al. [9]. The concentration of carbon dioxide was measured using a SRI 8610C gas chromatography (GC) with thermal conductivity (TC) detector. It was analyzed on a 3' Silica Gel column (column temperature: $105^{\circ} \mathrm{C}$ ) with helium as the carrier gas at 10 psia at oven temperature of $80{ }^{\circ} \mathrm{C}$.

\section{Results and discussion}

\subsection{Simultaneous complete consumption of glucose and xylose}

Among published co-culture studies, incomplete xylose consumption is one of the most common limitations due to the catabolite repression. In this study, this limitation is alleviated by feeding the mixed substrates into $S$. cerevisiae chamber. By controlling biomass densities of both strains, glucose concentration in the feed medium, and dilution rate, complete consumption of glucose can be achieved in the $S$. cerevisiae chamber, leaving only xylose reaches $S$. stipitis chamber. At the beginning of the experiment, due to the low cell density of $S$. cerevisiae, the amount of glucose consumed by the cells was smaller than the glucose fed into the reactor which caused glucose accumulation. However, as the cell density quickly picked up, the glucose consumption rate increased dramatically and eventually was limited by glucose feeding rate which resulted in the glucose completion and stayed at zero. In this way, the diauxic kinetics of S. stipitis often exhibited in continuous or fed-batch fermentations can be eliminated. The simultaneous complete 
utilization of both glucose and xylose was achieved in this study as shown in Figure 2 (a) (after 70 hours).

\subsection{Independent monitoring and control of biomass development}

Among published co-culture studies, the concentrations of individual biomass have not been reported, as two strains were mixed together after the inoculation and it was difficult to measure the individual biomass density in the mixture. With the membraneseparated bioreactor, it is easy to track the cell mass development of each strain during the course of the experiment. More importantly, the membrane-separated bioreactor with the dual pseudo-continuous/continuous operation enables the independent control of biomass development of each strain. In addition, the cell growth rate of S. stipitis was controlled through manipulating OUR in the chamber, as S. stipitis' growth rate is highly sensitive to the availability of oxygen. On the other hand, because $S$. cerevisiae is a crab-tree positive strain which can grow anaerobically, the cell grow rate of $S$. cerevisiae was controlled through the availability of substrate (glucose) by manipulating feeding concentration of substrate. Besides, the initial inoculation ratio of the two strains was optimized to enable a more balanced cell growth.

After several runs of trial-and-error, we found the following experimental condition is a robust way to achieve the desired cell mass development. The initial optical densities (OD) of both $S$. cerevisiae and $S$. stipitis were $1.0\left(\mathrm{OD}_{600}\right)$ after inoculation, with the initial culture medium in both chambers of the reactor being $20 \mathrm{~g}$ D-xylose with the standard nitrogen base solution. Instead of batch operation, cell growth stage was implemented using pseudo-continuous operation, with the feed-in medium containing $100 \mathrm{~g}$ D-glucose with the standard nitrogen base solution. In this way, we can control the cell growth rate of $S$. 
cerevisiae though manipulating substrate feed flow rate, while controlling the cell growth rate of $S$. stipitis through manipulating the amount of oxygen in the feeding gas. During fermentation stage, the feed-in medium switched to a mixture of glucose and xylose with the standard nitrogen base solution, and the biomass control can be achieved in the same way. A typical biomass development trajectory for both chambers is shown in Figure 2 (a).

\subsection{Independent control of oxygen condition}

With the membrane separating the two chambers, it is possible to maintain different oxygen conditions: anaerobic for S. cerevisiae while microaerobic for S. stipitis. In order to validate the independent control of different OUR conditions in both chambers, we measured the dissolved oxygen (DO) in the liquid phase and oxygen content in the offgas using the bioreactor without cells. It is worth noting that this cannot be done with cells because the cells consume oxygen. The experimental results were given in Figure 3 . The $\sim 3$ minutes $100 \%$ DO measurement of the off-gas from the $S$. cerevisiae chamber is the time needed for nitrogen gas to completely replace the mixed gas initially in the head space which contains oxygen and was calibrated as $100 \%$. Figure 3 clearly shows that different OUR conditions can be achieved: anaerobic in S. cerevisiae chamber (0\% DO) and microaerobic for S. stipitis (100\% DO).

\subsection{Controlled chemostat under different environmental factors}

The purpose of developing the bioreactor and related protocols is to conduct systematic investigations on the co-culture dynamics under chemostat, which would allow the quantitative study on the effect of different environmental factors. Among different factors such as population ratio, feeding concentration and dilution rate, OUR is arguably the most important one, as ethanol production by S. stipitis is highly sensitive to OUR. The 
conventional feedback control module equipped with many commercial bioreactors is not sufficient to achieve stable OUR, as it relies on the DO measurement to determine how much oxygen (air) to be fed into the reactor. For S. stipitis, once the biomass density reaches $3 \mathrm{gDCW} / \mathrm{L}$, oxygen transfer becomes diffusion limited and the DO measurement stays at zero all the time which makes the conventional DO-based feedback control ineffective.

In this work stable OUR was achieved through maintaining constant biomass and constant oxygen supply. The dual continuous/pseudo-continuous operation allows the easy maintenance of constant cell density under much wider oxygen conditions, particularly at lower OUR ranges. Figure 4 shows the data from one experiment where chemostat under different OUR conditions were achieved. Among different conditions tested in our experiments, ethanol yield and conversion rates varies in the range of $0.12-0.44 \mathrm{~g} / \mathrm{g}$ and 0.22-1.95 $\mathrm{gEtOH} / \mathrm{L} / \mathrm{hr}$, respectively, which are in line with the maximum ethanol yield and conversion rate reported in the literature as listed in Table 1. As shown in Table 1, batch operations count the vast majority of co-culture studies in recent publications, with the only continuous operation using a different strain pair (Z. mobilis and S. stipitis). To make the comparison more relevant, we listed results from two older publications (i.e., [26] and [27] in Table 1), which reported the best ethanol yield of 0.42 and $0.33 \mathrm{~g} / \mathrm{g}$, and best productivity of 0.075 and $0.43 \mathrm{gEtOH} / \mathrm{L} / \mathrm{hr}$, respectively. It is worth noting that the main objective of this work is to develop new equipment, procedures, and control strategies that can deliver controlled, continuous chemostat operation in order to facilitate systematic study of the co-culture system, instead of maximizing the ethanol yield or productivity using the co-culture system. 


\subsection{Improvement on ethanol tolerance under pseudo-continuous fermentation}

The accumulation of ethanol during cultivation causes stress to yeast cells [19], which drives the cells to adjust their intracellular physiological conditions to the surrounding environment in order to grow [20]. One added benefit of the pseudocontinuous operation is that it provides an ideal environment for cells to adapt to higher ethanol conditions, as the environmental pressure is consistently applied and gradually increases. To verify this, we sampled cells from the bioreactor after 2 weeks of experiment, and tested the cells viability after 2 hours ethanol shock with various ethanol concentrations, and compared with that of unadapted cells. Figure 5 shows the ethanol tolerance tests performed on both unadapted and adapted cells of S. cerevisiae and S. stipitis with glucose and xylose as carbon sources, respectively. The ethanol tolerance of adapted cells of both $S$. cerevisiae and S. stipitis were noticeably improved by adaptation during relatively short (2 weeks) pseudo-continuous fermentation. The cell viability difference between the unadapted and adapted cells increases as ethanol concentration increases, particularly at higher ethanol concentrations of $90 \mathrm{~g} / \mathrm{L}$ for $S$. cerevisiae and $60 \mathrm{~g} / \mathrm{L}$ for S. stipitis. In those two cases, the survival rates increased from $36 \%$ to $88 \%$ for $S$. cerevisiae and from $13 \%$ to $45 \%$ for S. stipitis. It was surprising to observe the further improvement on ethanol tolerance of $S$. cerevisiae as it already has much higher ethanol tolerance compared to $S$. stipitis. The improved ethanol tolerance is likely due to physiological changes as cells adapt to gradually increased ethanol concentration while they grow, instead of genetic mutations, as they lost such improved tolerance after two rounds of plate transfer. However, such physiological change will be maintained as the environmental pressure is always there during chemostat operation. 


\section{Conclusion}

The co-culture system of $S$. cerevisiae and $S$. stipitis has drawn increasing interest in the last few years to ferment the mixture of glucose and xylose or lignocellulosic hydrolysate. However, existing research mainly examined the ethanol production performance of different co-culture systems such as final ethanol yield and productivity, with little or no attempt to understand the dynamic interaction between the two microbes. In this work, we developed a membrane-separated bioreactor and associated protocols and control strategies to facilitate quantitative study of co-culture systems. Through the inhouse developed cell retention module and gas mixing apparatus, as well as the dual-mode continuous/pseudo-continuous operation, we were able to not only independently monitor and control biomass development of each strain, but also to independently control different OUR conditions for each strain. As a result, chemostat operations of the co-culture system were achieved under various conditions, particularly at lower OUR conditions. To the best of our knowledge, this work is the first report of achieving chemostat operation of coculture or single culture of $S$. stipitis with stably controlled OUR. Specifically, no published research reported the measurement, not to mention the control, of OUR during experiments. In addition, the proposed system enables easy monitoring and control of the cell mass development of each strain independently after inoculation, which has not been achieved in co-culture studies before. Specifically, none of the existing co-culture studies reported the tracking, not to mention control, of individual strain population during experiments. Only the initial inoculum concentrations of each strain were provided. It is worth noting that the developed bioreactor, its operation protocols and control strategies can be adapted to the study of other co-culture systems. 


\section{Acknowledgment}

Financial supports from USDA-AFRI (2010-65504-20358), Sun Grant. (8500014161), and NSF $(1264861,1248388)$ are gratefully acknowledged. The authors also would like to thank Dr. Thomas W. Jeffries (University of Wisconsin-Madison) for helpful discussions. 


\section{References}

[1] T.W. Jeffries, Y.-S. Jin, Metabolic engineering for improved fermentation of pentoses by yeasts, Appl. Microbiol. Biotechnol. 63 (2004) 495-509.

[2] M. Kuyper, M.J. Toirkens, J.A. Diderich, A.A. Winkler, J.P. Dijken, J.T. Pronk, Evolutionary engineering of mixed-sugar utilization by a xylose-fermenting Saccharomyces cerevisiae strain, FEMS Yeast Res. 5 (2005) 925-934.

[3] M. Ma, Z.L. Liu, J. Moon, Genetic engineering of inhibitor-tolerant Saccharomyces cerevisiae for improved xylose utilization in ethanol production, BioEnergy Res. 5 (2012) 459-469.

[4] D.R.J. Grootjen, L.H.H.M. Meijlink, R.G.J.M. Van der Lans, K.C.A. Luyben, Cofermentation of glucose and xylose with immobilized Pichia stipitis and Saccharomyces cerevisiae, Enzym. Microb. Technol. 12 (1990) 860-864.

[5] D.R.J. Grootjen, L.H.H.M. Meijlink, R. Vleesenbeek, R.G.J.M. Van der Lans, K.C.A. Luyben, Cofermentation of glucose and xylose with immobilized Pichia stipitis in combination with Saccharomyces cerevisiae, Enzym. Microb. Technol. 13 (1991) 530-536. [6] T.J. Hanly, M. a. Henson, Dynamic flux balance modeling of microbial co-cultures for efficient batch fermentation of glucose and xylose mixtures, Biotechnol. Bioeng. 108 (2011) 376-85.

[7] J. Delgenes, R. Moletta, J. Navarro, The ethanol tolerance of Pichia stipitis Y 7124 grown on a d-xylose, d-glucose and 1-arabinose mixture, J. Ferment. Technol. 66 (1988) 417-422.

[8] H.N. Chang, I.-K. Yoo, B.S. Kim, High density cell culture by membrane-based cell recycle, Biotechnol. Adv. 12 (1994) 467-487.

[9] M. Taniguchi, Ethanol production from a mixture of glucose and xylose by a novel co-culture system with two fermentors and two microfiltration modules, J. Ferment. Bioeng. 84 (1997) 59-64.

[10] L. Slomi’nska, W. Grajek, A. Grze'skowiak, M. Gocalek, Enzymatic starch saccharification in an ultrafiltration membrane reactor, Starch-Stärke. 50 (1998) 390-396.

[11] G.M. Rios, M.P. Belleville, D. Paolucci, J. Sanchez, Progress in enzymatic membrane reactors - a review, J. Membr. Sci. 242 (2004) 189-196.

[12] S. Yang, W. Ding, H. Chen, Enzymatic hydrolysis of rice straw in a tubular reactor coupled with UF membrane, Process. Biochem. 41 (2006) 721-725.

[13] M. Liang, M.H. Kim, Q.P. He, J. Wang, Impact of pseudo-continuous fermentation on the ethanol tolerance of Scheffersomyces stipitis, J. Biosci. Bioeng. 116 (2013) 319-326. [14] M. Rizzi, C. Klein, C. Schulze, N.A. Bui-Thanh, H. Dellweg, Xylose fermentation by yeasts. 5. Use of ATP balances for modeling oxygen-limited growth and fermentation of yeast Pichia stipitis with xylose as carbon source., Biotechnol. Bioeng. 34 (1989) 509-514. [15] J.C. du Preez, Process parameters and environmental factors affecting d-xylose fermentation by yeasts, Enzym. Microb. Technol. 16 (1994) 944-956.

[16] P.J. Slininger, L.E. Branstrator, R.J. Bothast, M.R. Okos, M.R. Ladisch, Growth, death, and oxygen uptake kinetics of Pichia stipitis on xylose, Biotechnol. Bioeng. 37 (1991) 973-80.

[17] A.L. Damiani, M.H. Kim, J. Wang, An improved dynamic method to measure kLa in bioreactors, Biotechnol. Bioeng. 111 (2014) 2120-2125. 
[18] B. Zhou, G.J. Martin, N.B. Pamment, Increased phenotypic stability and ethanol tolerance of recombinant Escherichia coli KO11 when immobilized in continuous fluidized bed culture, Biotechnol. Bioeng. 100 (2008) 627-633.

[19] P.V. Attfield, Stress tolerance: the key to effective strains of industrial baker's yeast, Nat. Biotechnol. 15 (1997) 1351-1357.

[20] T.N. Dinh, K. Nagahisa, T. Hirasawa, C. Furusawa, H. Shimizu, Adaptation of Saccharomyces cerevisiae cells to high ethanol concentration and changes in fatty acid composition of membrane and cell size., PloS One. 3 (2008).

[21] B. Gutiérrez-Rivera, K. Waliszewski-Kubiak, O. Carvajal-Zarrabal, M.G. AguilarUscanga, Conversion efficiency of glucose/xylose mixtures for ethanol production using Saccharomyces cerevisiae ITV01 and Pichia stipitis NRRL Y-7124, J. Chem. Technol. \& Biotechnol. 87 (2012) 263-270.

[22] B. Gutiérrez-Rivera, B. Ortiz-Muñiz, J. Gómez-Rodríguez, A. Cárdenas-Cágal, J.M.D. González, M.G. Aguilar-Uscanga, Bioethanol production from hydrolyzed sugarcane bagasse supplemented with molasses "B" in a mixed yeast culture, Renew. Energy. 74 (2015) 399-405.

[23] G. Chaudhary, S. Ghosh, Two-reactor, continuous culture fermentation for fuel ethanol production from lignocellulosic acid hydrolysate using Zymomonas mobilis and Scheffersomyces stipitis, RSC Adv. 4 (2014).

[24] L.R. Hickert, P.B. de Souza-Cruz, C.A. Rosa, M.A.Z. Ayub, Simultaneous saccharification and co-fermentation of un-detoxified rice hull hydrolysate by Saccharomyces cerevisiae ICV D254 and Spathaspora arborariae NRRL Y-48658 for the production of ethanol and xylitol, Bioresour. Technol. 143 (2013) 112-116.

[25] L.R. Hickert, F. da Cunha-Pereira, P.B. de Souza-Cruz, C.A. Rosa, M.A.Z. Ayub, Ethanogenic fermentation of co-cultures of Candida shehatae HM 52.2 and Saccharomyces cerevisiae ICV D254 in synthetic medium and rice hull hydrolysate, Bioresour. Technol. 131 (2013) 508-514.

[26] A. Singh, N.R. Bishnoi, Enzymatic hydrolysis optimization of microwave alkali pretreated wheat straw and ethanol production by yeast, Bioresour. Technol. 108 (2012) 94-101.

[27] A.K. Chandel, O.V. Singh, M.L. Narasu, L.V. Rao, Bioconversion of Saccharum spontaneum (wild sugarcane) hemicellulosic hydrolysate into ethanol by mono and cocultures of Pichia stipitis NCIM3498 and thermotolerant Saccharomyces cerevisiae-VS $\mathrm{V}_{3}$, New Biotechnol. 28 (2011) 593-599.

[28] F. da Cunha-Pereira, L.R. Hickert, N.T. Sehnem, P.B. de Souza-Cruz, C.A. Rosa, M.A.Z. Ayub, Conversion of sugars present in rice hull hydrolysates into ethanol by Spathaspora arborariae, Saccharomyces cerevisiae, and their co-fermentations., Bioresour. Technol. 102 (2011) 4218-4225.

[29] Y. Li, J. Park, R. Shiroma, K. Tokuyasu, Bioethanol production from rice straw by a sequential use of Saccharomyces cerevisiae and Pichia stipitis with heat inactivation of Saccharomyces cerevisiae cells prior to xylose fermentation., J. Biosci. Bioeng. 111 (2011) 682-686.

[30] K.S. Yadav, S. Naseeruddin, G.S. Prashanthi, L. Sateesh, L.V. Rao, Bioethanol fermentation of concentrated rice straw hydrolysate using co-culture of Saccharomyces cerevisiae and Pichia stipitis., Bioresour. Technol. 102 (2011) 6473-6478. 
[31] J.M. Laplace, J.P. Delgenes, R. Moletta, J.M. Navarro, Cofermentation of glucose and xylose to ethanol by a respiratory-deficient mutant of Saccharomyces cerevisiae cocultivated with a xylose-fermenting yeast, J. Ferment. Bioeng. 75 (1993) 207-212.

[32] D.R.J. Grootjen, M.L. Jansen, R.G.J.M. van der Lans, K.C.A.M. Luyben, Reactors in series for the complete conversion of glucose/xylose mixtures by Pichia stipitis and Saccharomyces cerevisiae, Enzym. Microb. Technol. 13 (1991) 828-833. 


\section{Figure Captions}

Figure 1. (a) The schematic diagram of co-culture system; (b) Actual set-up of the developed bioreactor for proposed co-culture system

Figure 2. (a) Biomass development of $S$. cerevisiae and S. stipitis, and simultaneous consumption of glucose and xylose; (b) Concentrations of ethanol and byproducts of $S$. cerevosiase and S. stipitis

Figure 3. Separate control of DO in co-culture system; DO measurement in liquid (top); DO measurement in offgas (bottom), Feed only $\mathrm{N}_{2}$ into $S$. cerevisiae and mixture of Air (172 $\mathrm{mL} / \mathrm{min})$ and $\mathrm{N}_{2}(26 \mathrm{~mL} / \mathrm{min})$ into S. stipitis.

Figure 4. Constant biomass of S. cerevisiae and S. stipitis (top); Constant OUR of S. stipitis under controlled chemostat

Figure 5. Cell viability of adapted and unadapted strains of $S$. cerevisiae under ethanol shock (top); Cell viability of adapted and unadapted strains of S. stipitis under ethanol shock (bottom) 
Table 1. Recent work in ethanol production using co-culture systems (with two older continuous studies)

\begin{tabular}{|c|c|c|c|c|c|}
\hline Reference & Co-culture strains & $\begin{array}{l}\text { Operation } \\
\text { mode }\end{array}$ & $\begin{array}{l}\text { Best theoretical } \\
\text { yield }(\%)\end{array}$ & $\begin{array}{l}\text { Best } \\
\text { yield } \\
(\mathrm{g} / \mathrm{g})\end{array}$ & $\begin{array}{l}\text { Best } \\
\text { productivity } \\
(\mathrm{g} / \mathrm{L} / \mathrm{hr})\end{array}$ \\
\hline$[21,22]$ & S. cerevisiae - S. stipitis & batch & $\begin{array}{l}78 \% \text { at } 200 \mathrm{hr} \\
88 \% \text { at } 42 \mathrm{hr}\end{array}$ & $\begin{array}{l}0.40 \\
0.45\end{array}$ & $\begin{array}{l}1.26 \\
1.07\end{array}$ \\
\hline [23] & $\begin{array}{l}\text { Z. mobilis - S. stipitis } \\
\text { (reactors in series) }\end{array}$ & continuous & $\begin{array}{l}92 \% \text { for } Z . \text { mobilis } \\
\text { with dilution rate } \\
0.07 \mathrm{hr}^{-1} \text { at } 168 \mathrm{hr} \\
88 \% \text { for } S . \text { stipitis } \\
\text { with dilution rate } \\
0.048 \mathrm{hr}^{-1} \text { at } 168 \mathrm{hr}\end{array}$ & 0.47 & 2.20 \\
\hline$[24,25]$ & $\begin{array}{l}\text { S. cerevisiae }- \text { S. arborariae } \\
\text { S. cerevisiae }- \text { C. shehatae }\end{array}$ & batch & $\begin{array}{l}94 \% \text { at } 250 \mathrm{hr} \\
94 \% \text { at } 250 \mathrm{hr}\end{array}$ & $\begin{array}{l}0.48 \\
0.48\end{array}$ & $\begin{array}{l}0.11 \\
0.15\end{array}$ \\
\hline [26] & S. cerevisiae - S. stipitis & batch & $78 \%$ at $36 \mathrm{hr}$ & 0.40 & 0.49 \\
\hline [27] & S. cerevisiae -S. stipitis & batch & $94 \%$ at $84 \mathrm{hr}$ & 0.48 & 0.22 \\
\hline [28] & S. cerevisiae - S. arborariae & batch & $75 \%$ at $150 \mathrm{hr}$ & 0.38 & 0.25 \\
\hline [29] & $\begin{array}{l}\text { S. cerevisiae }- \text { S. stipitis } \\
\text { (inactivate } S . \text { cerevisiae) }\end{array}$ & batch & $85 \%$ at $125 \mathrm{hr}$ & 0.43 & 0.12 \\
\hline [30] & S. cerevisiae -S. stipitis & batch & $78 \%$ at $36 \mathrm{hr}$ & 0.40 & 0.33 \\
\hline [31] & $\begin{array}{l}\text { (RD) S. cerevisiae - S. } \\
\text { stipitis }\end{array}$ & continuous & $82 \%$ at $240 \mathrm{hr}$ & 0.42 & 0.075 \\
\hline [32] & $\begin{array}{l}\text { S. cerevisiae }-S \text {. stipitis } \\
\text { (reactors in series) }\end{array}$ & continuous & $64 \%$ at $816 \mathrm{hr}$ & 0.33 & 0.43 \\
\hline
\end{tabular}




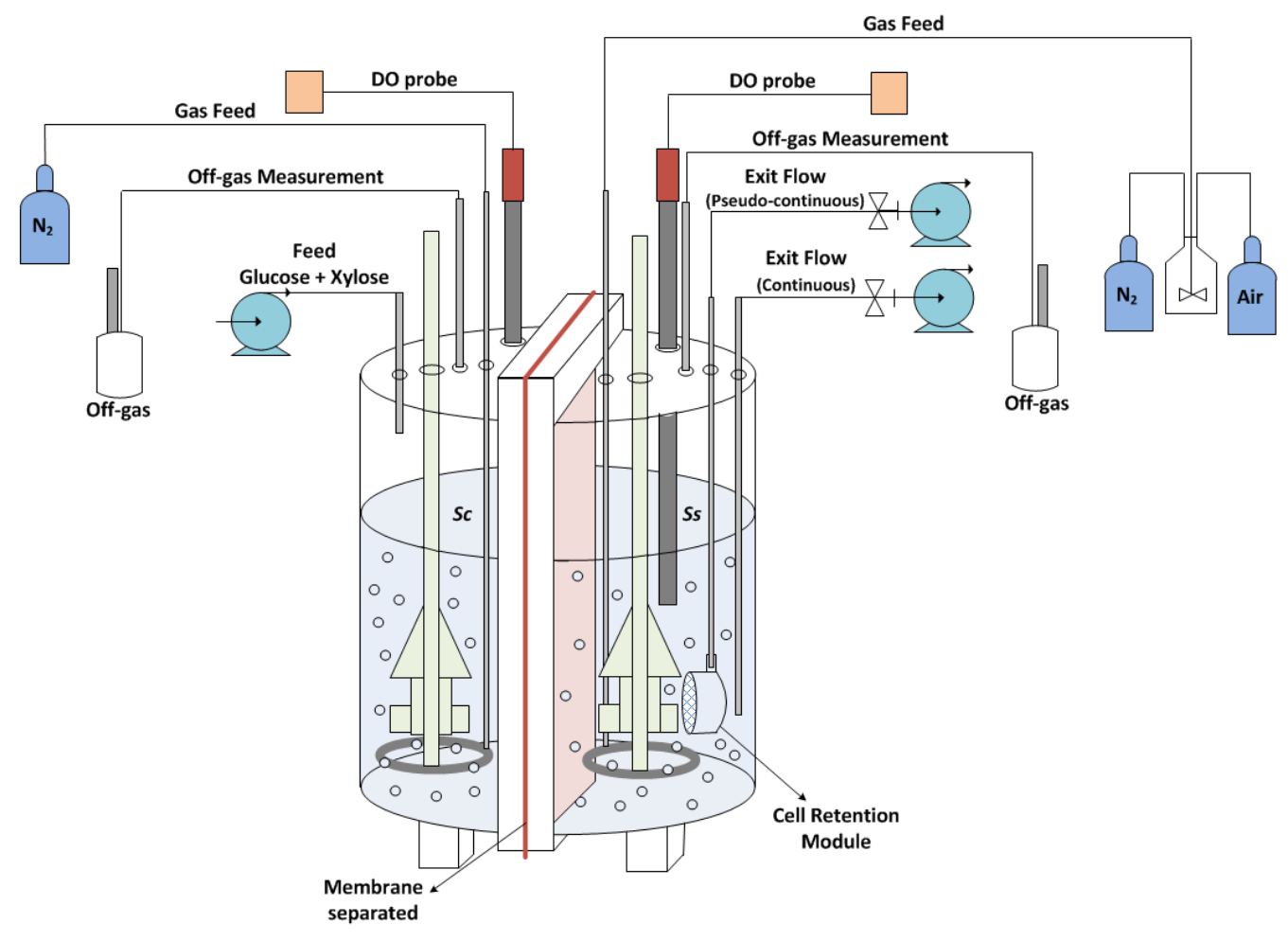

(a)

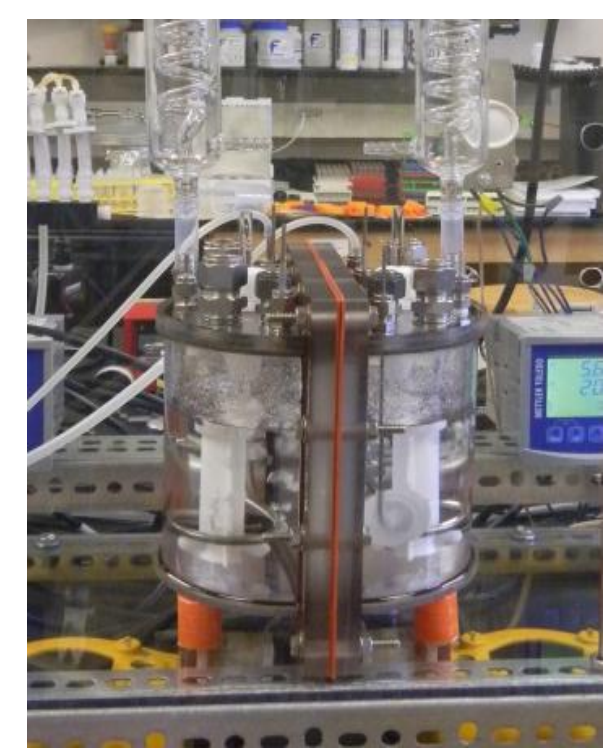

(b)

Figure 1. (a) The schematic diagram of co-culture system; (b) Actual set-up of the developed bioreactor for proposed co-culture system 


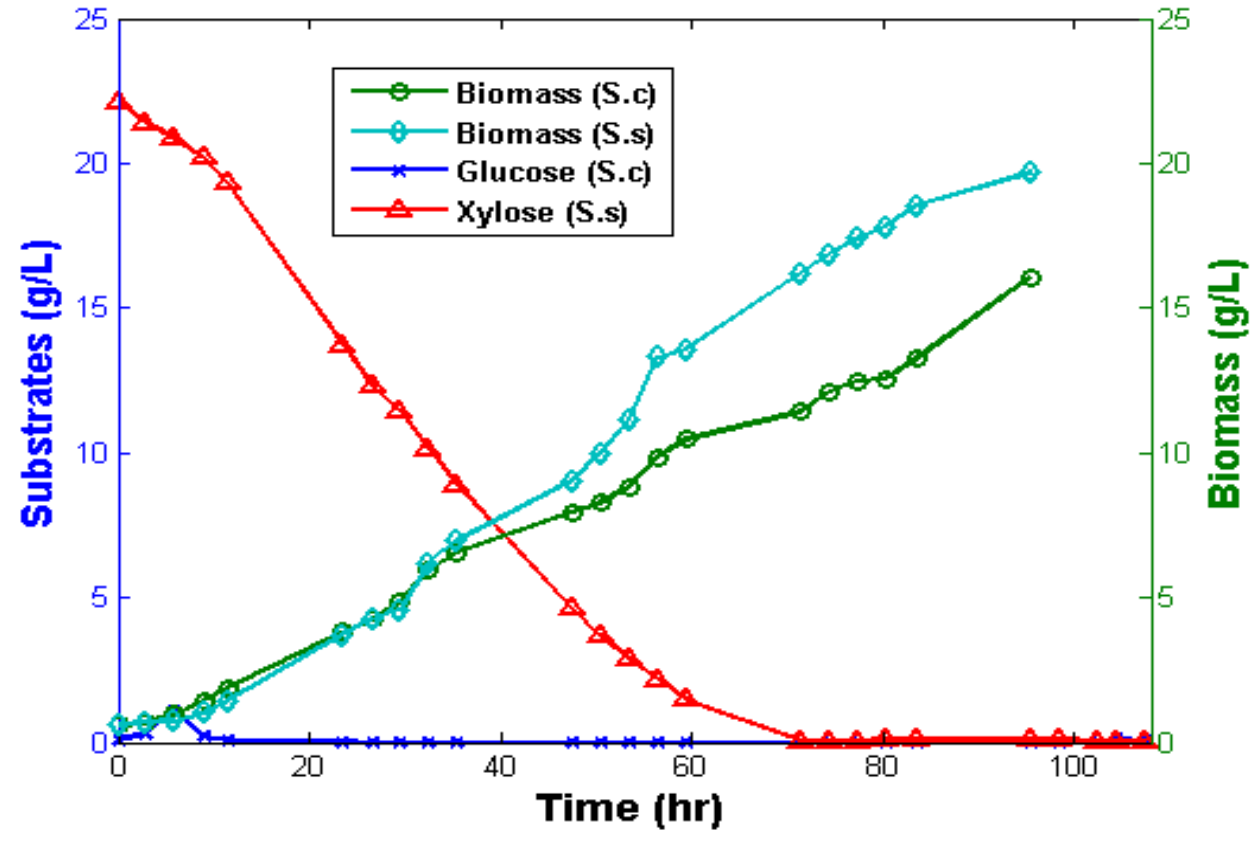

(a)
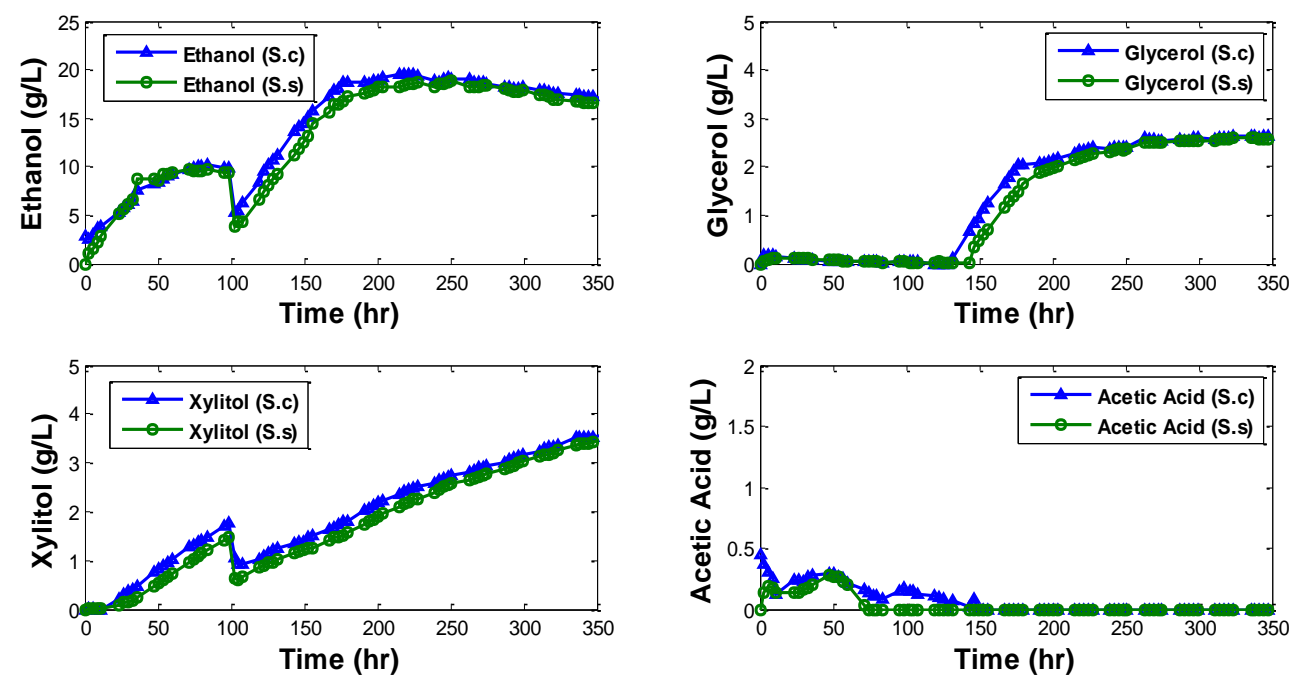

(b)

Figure 2. (a) Biomass development of $S$. cerevisiae and $S$. stipitis, and simultaneous consumption of glucose and xylose ; (b) Concentrations of ethanol and byproducts of $S$. cerevisiae and S. tipitis. (S.c) in the legend indicates $S$. cerevisiae chamber while (S.s) indicates $S$. stipitis chamber. 

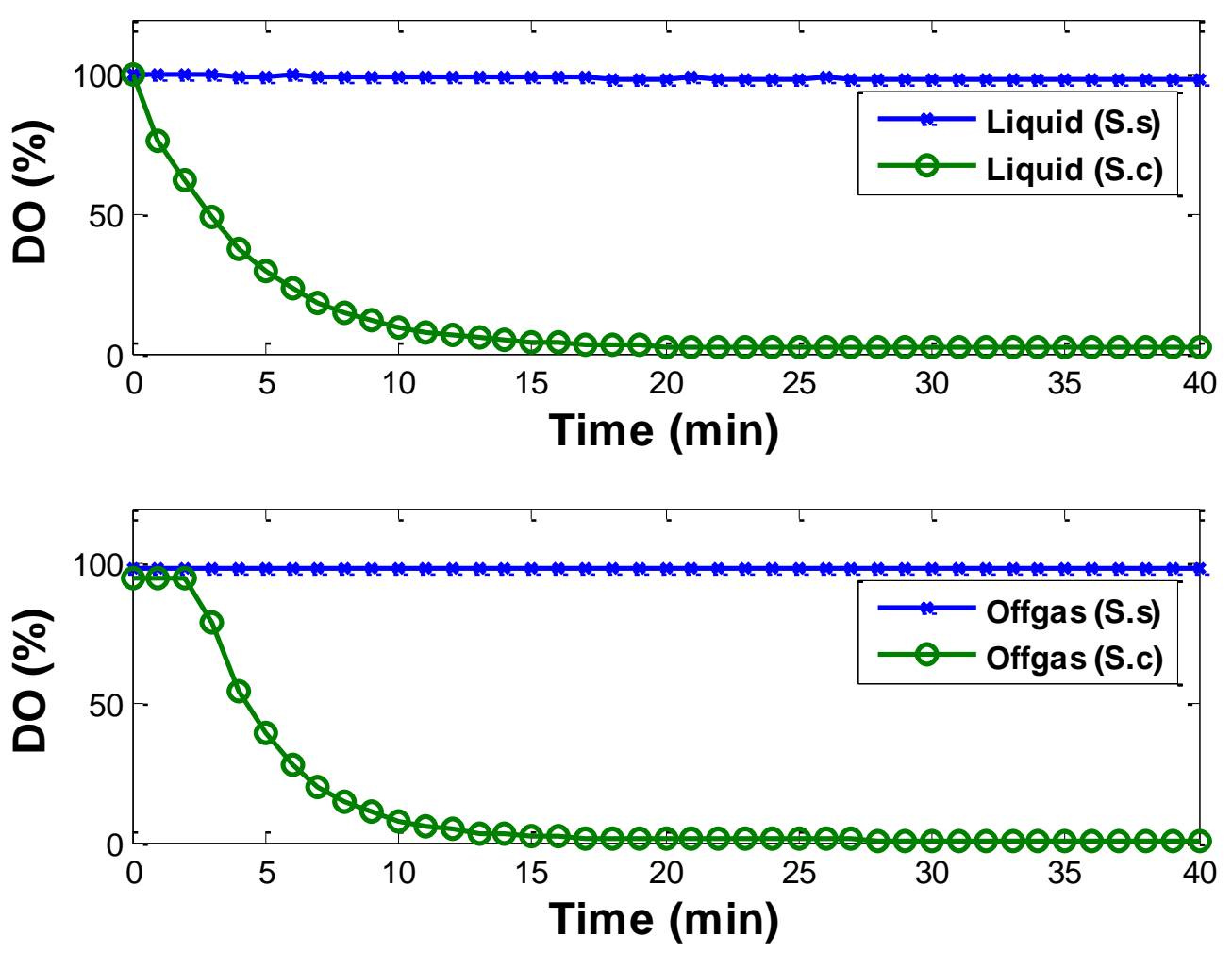

Figure 3. Separate control of DO in co-culture system; DO measurement in liquid (top); DO measurement in offgas (bottom), Feed only $\mathrm{N}_{2}$ into $S$. cerevisiae and mixture of Air $(172 \mathrm{~mL} / \mathrm{min})$ and $\mathrm{N}_{2}(26 \mathrm{~mL} / \mathrm{min})$ into $S$. stipitis 

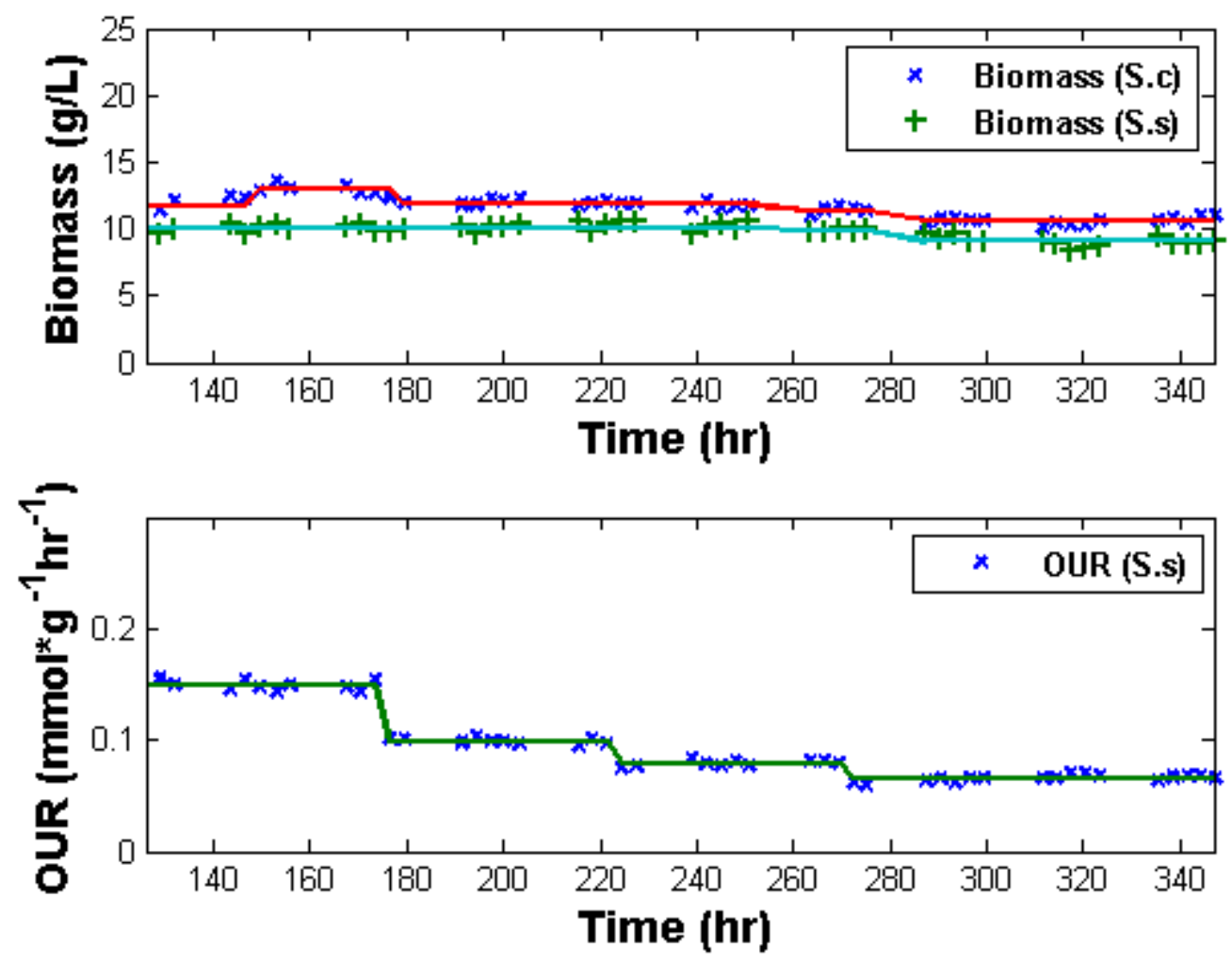

Figure 4. Constant biomass of $S$. cerevisiae and $S$. stipitis (top); Constant OUR of $S$. stipitis under controlled chemostat (bottom) 

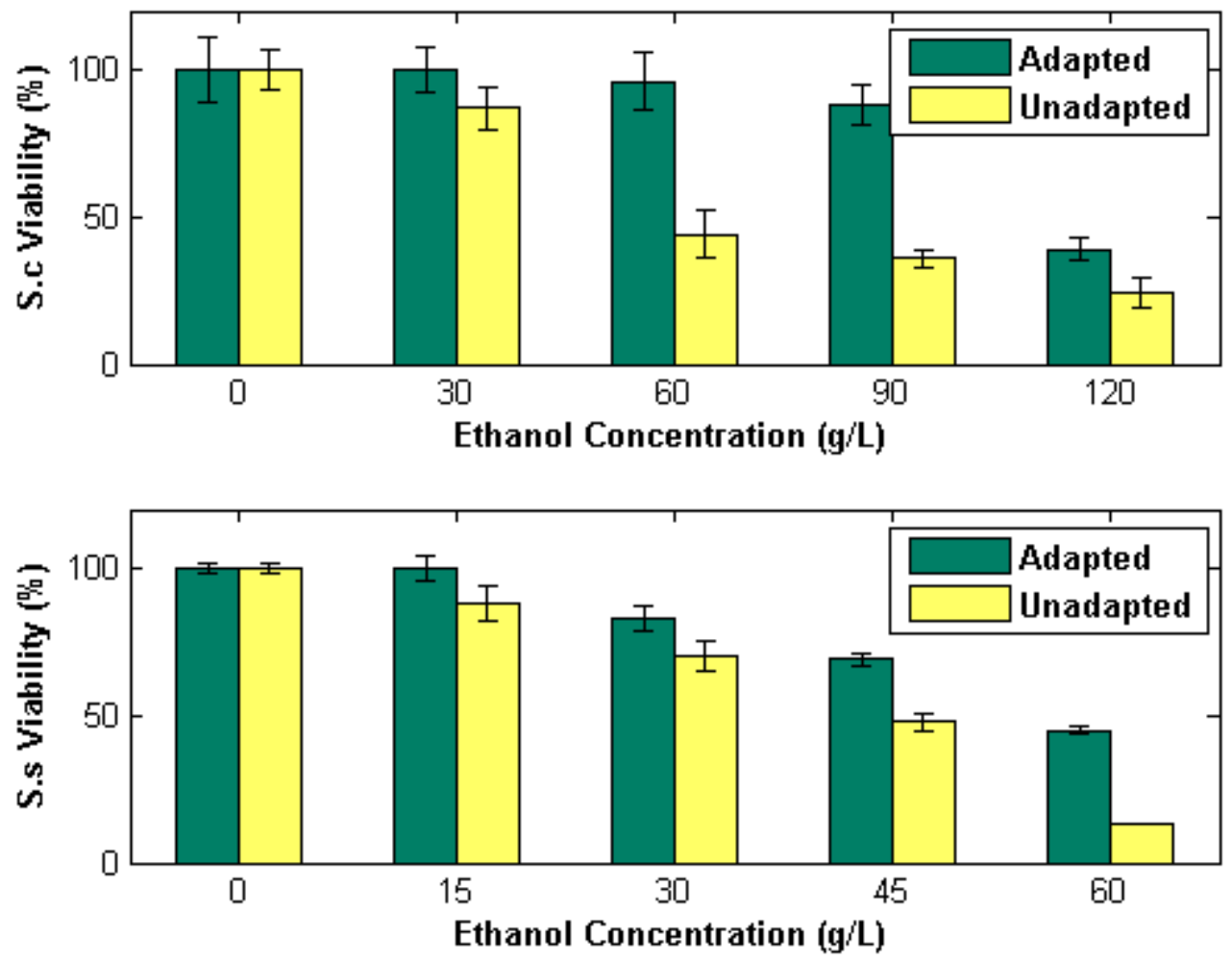

Figure 5. Cell viability of adapted and unadapted strains of $S$. cerevisiae under ethanol shock (top); Cell viability of adapted and unadapted strains of S. stipitis under ethanol shock (bottom) 


\section{Appendix A}

1. Mass balance of biomass development under pseudo-continuous operation:

$$
\frac{d X}{d t}=\mu X
$$

where $X$ is the biomass concentration $(g / L), t$ the time $(h r)$, and $\mu$ the cell growth rate $\left(h r^{-1}\right)$.

2. Mass balance of biomass development under continuous operation:

$$
\frac{d X}{d t}=\left(\mu-\frac{F}{V}\right) X
$$

where $F$ is the feed flow rate $(L / h r)$, and $V$ the volume $(L)$.

3. Calculation for operation durations:

The biomass profile during the dual continuous/pseudo-continuous operation is illustrated in Figure 6.

First, we calculate cell accumulation during the pseudo-continuous operation based on Eqn. (1):

$$
X_{1}=X_{0} e^{\mu t_{1}}
$$

where $X_{0}$ and $X_{1}$ are the biomass concentrations at the beginning and end of the pseudo-continuous operation, respectively; $t_{1}$ the duration of the pseudo-continuous operation. 
Then, we calculate cell loss during the continuous operation following the pseudocontinuous operation based on Eqn. (2):

$$
X_{2}=X_{1} e^{\left(\mu-\frac{F}{V}\right)\left(t-t_{1}\right)}
$$

where $X_{1}$ and $X_{2}$ are the biomass concentrations at the beginning and end of the continuous operation, respectively. $t$ is the duration of both operations, which can be set as $24 \mathrm{hrs}$ for convenience, and $\left(t-t_{1}\right)$ is the duration of the continuous operation.

Finally, to maintain biomass concentration at constant, we set the final concentration to be the same as the initial concentration for the period of $t$ :

$$
X_{0}=X_{2}
$$

and we use $t_{2}$ to represent the duration of the continuous operation:

$$
t_{2}=t-t_{1}
$$

plugging Eqns. (3), (4) and (6) into Eqn. (5), we have

$$
X_{0}=X_{0} e^{\mu t_{1}+\left(\mu-\frac{F}{V}\right) t_{2}}
$$

therefore,

$$
\mu t_{1}+\left(\mu-\frac{F}{V}\right) t_{2}=0
$$

and we have, 


$$
\frac{t_{1}}{t_{2}}=\frac{\left(\frac{F}{V}-\mu\right)}{\mu}
$$

or

$$
\frac{t_{1}}{t_{2}}=\frac{(D-\mu)}{\mu}
$$

where $D=\frac{F}{V}$ is the dilution rate $\left(h r^{-1}\right)$.

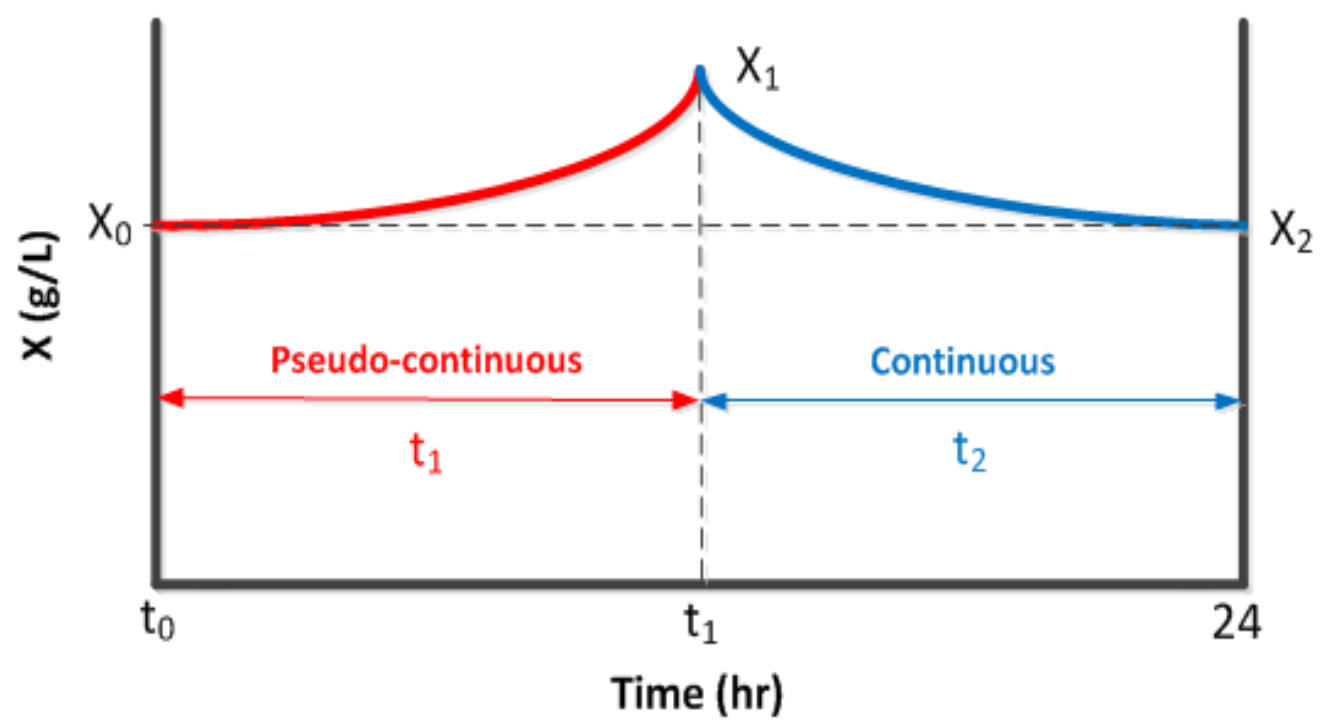

Figure 6. Illustration of biomass profile during the dual continuous/pseudo-continuous operation 